\title{
Nanoscale
}

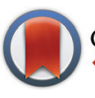

CrossMark

\& click for update

Cite this: Nanoscale, 2015, 7, 7234

Received 10th January 2015,

Accepted 12th March 2015

DOI: 10.1039/c5nr00194c

www.rsc.org/nanoscale

\section{Anti-epidermal growth factor receptor conjugated mesoporous zinc oxide nanofibers for breast cancer diagnostics $\uparrow$}

\author{
Md. Azahar Ali, $\neq^{\mathrm{a}}$ Kunal Mondal, $\neq^{\mathrm{a}}$ Chandan Singh, ${ }^{\mathrm{b}}$ Bansi Dhar Malhotra*c and \\ Ashutosh Sharma*a
}

We report the fabrication of an efficient, label-free, selective and highly reproducible immunosensor with unprecedented sensitivity (femto-molar) to detect a breast cancer biomarker for early diagnostics. Mesoporous zinc oxide nanofibers ( $\mathrm{ZnOnFs)}$ ) are synthesized by electrospinning technique with a fiber diameter in the range of 50-150 nm. Fragments of ZnOnFs are electrophoretically deposited on an indium tin oxide glass substrate and conjugated via covalent or electrostatic interactions with a biomarker (antiErbB2; epidermal growth factor receptor 2). Oxygen plasma treatment of the carbon doped ZnOnFs generates functional groups $(-\mathrm{COOH},-\mathrm{OH}$, etc.) that are effective for the conjugation of anti-ErbB2. ZnOnFs without plasma treatment that conjugate via electrostatic interactions were also tested for comparison. Label-free detection of the breast cancer biomarker by this point-of-care device is achieved by an

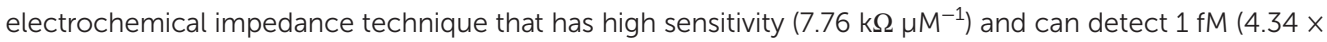
$10^{-5} \mathrm{ng} \mathrm{mL}{ }^{-1}$ ) concentration. The excellent impedimetric response of this immunosensor provides a fast detection (128 s) in a wide detection test range (1.0 fM-0.5 $\mu \mathrm{M})$. The oxy-plasma treated ZnOnF immunoelectrode shows a higher association constant $\left(404.8 \mathrm{kM}^{-1} \mathrm{~s}^{-1}\right)$ indicating a higher affinity towards the ErbB2 antigen compared to the untreated $\mathrm{ZnOnF} \mathrm{immunoelectrode}\left(165.6 \mathrm{kM}^{-1} \mathrm{~s}^{-1}\right)$. This sensor is about an order of magnitude more sensitive than the best demonstrated in the literature based on different nanomaterials and about three orders of magnitude better than the ELISA standard for breast cancer biomarker detection. This proposed point-of-care cancer diagnostic offers several advantages, such as higher stability, rapid monitoring, simplicity, cost-effectiveness, etc., and should prove to be useful for the detection of other bio- and cancer markers.
\end{abstract}

\section{Introduction}

With recent advances in genomics, proteomics and molecular pathology, the detection of cancer biomarkers for the investigation of genetic changes during malignant transformations is found to be an interesting tool. ${ }^{1}$ Cancer biomarkers are the chemical substances that are related to malignant tumors and can be found in blood, urine, stool, other bodily fluids, or tissues of affected patients. ${ }^{2,3}$ Growth factor receptors, peptide

\footnotetext{
${ }^{a}$ Department of Chemical Engineering, Indian Institute of Technology, Kanpur, India. E-mail: ashutos@iitk.ac.in

${ }^{b}$ Department of Science and Technology Centre on Biomolecular Electronics, Biomedical Instrumentation Section, CSIR-National Physical Laboratory, New Delhi, India

${ }^{c}$ Department of Biotechnology, Delhi Technological University, Delhi, India. E-mail: bansi.malhotra@gmail.com

$\dagger$ †lectronic supplementary information (ESI) available. See DOI: 10.1039/ c5nr00194c

$\$$ These authors have contributed equally.
}

growth factors, signal transduction factors, tyrosine kinase and transcription-regulatory proteins are over-expressed in cancer cells. In particular, the human epidermal growth factor receptor (EGFR or ErbB2) has been found in around 33\% of breast cancers. ${ }^{4,5}$ The ErbB2 gene is found on the $17 \mathrm{q}$ chromosome and encodes for the $185 \mathrm{kDa}$ transmembrane tyrosine kinase growth factor receptor. ${ }^{6}$ The discovery of ErbB2 gene amplification, its overexpression in primary breast cancer and most importantly its association with a more aggressive clinical behavior makes ErbB2 a highly suitable candidate for diagnostics application.

The most widespread tools for breast cancer detection are $\mathrm{X}$-ray mammography and ultrasounds to investigate density changes in the breast. ${ }^{7}$ Although the magnetic resonance imaging technique is a highly sensitive tool, it lacks specificity. Besides this, early diagnostics and costs are major concerns for the quantification of cancerous molecules. Thus, biosensor devices $^{8}$ are considered to play an important role in the early detection of breast cancer molecules since they offer low costs, 
fast detection, portability, absence of side-effects, etc. It is known that the antigen and antibody of the epidermal growth factor receptor in a cancer cell provides specific biding sites (paratope and epitope) that allow the development of biosensor devices to detect cancerous molecules. ${ }^{9}$ For example, antibody conjugated quantum dots have been utilized for the detection of the HER2 biomarker in lung and breast cancer cells, ${ }^{8}$ and Yong et al. fabricated bioconjugated indium phosphide (core)-zinc sulfide (shell) quantum dot nanoprobes for the quantification of cancer molecules via imaging techniques. ${ }^{10}$ Akter et al. developed an electrochemical immunosensor based on multi walled carbon nanotubes and gold nanocomposites for the amplified detection of a prostate specific antigen. ${ }^{9}$

The application of nanostructured materials has grown rapidly in biomedical fields including molecular imaging, in vitro cancer cell studies, early diagnostics and personalized treatments of cancer. ${ }^{11}$ One dimensional (1D) nanorods, nanowires and nanofibers of zinc oxide $(\mathrm{ZnO})$ have been predicted to have considerable potential for biomedical applications including DNA delivery, bioimaging, magnetic resonance imaging, nanoelectronics and biosensors devices due to their higher surface-to-volume ratio, excellent electron transfer communication, environmental safety, biocompatibility, nontoxicity, chemical stability and biodegradability. ${ }^{12-16}$ The anisotropic structure of $\mathrm{ZnO}$ is known to exhibit higher electron mobility and diffusion co-efficient compared to other metal oxides like titanium oxide. ${ }^{17}$ Nanofibers of $\mathrm{ZnO}$ due to their high surface-to-volume and aspect ratios can play an important role in electrochemical biosensors. ${ }^{13}$ Zinc oxide nanofibers (ZnOnFs) may provide an electrical conduction path between proteins and electrodes that enhance charge transfer properties resulting in a higher sensitivity of the biodevices. Thus, there has been a rapid growth in the literature on the application of nanopore metal oxides, including $\mathrm{ZnO}$, for the detection of various biomolecules, such as glucose, DNA, antibodies and bacteria, with improved stability and selectivity. ${ }^{18}$ The nanopore structures result in an improved analytical performance due to the higher loading of active catalysts and higher diffusion rates. ${ }^{19}$ Nanopore $\mathrm{ZnO}$ is found to be the most stable because of its highly cross-linked structure that can resist biodegradation. $^{20}$ Thus, the pore size, shape, hydrophilicity/ hydrophobicity, charge distribution, $\mathrm{pH}$ environment and conductivity of ZnOnFs for biosensor applications can be tuned by varying the composition of the material during its synthesis process.

The bioconjugation of protein molecules with a metal oxide surface has recently attracted much interest the development of point-of-care (POC) diagnostics devices. ${ }^{21}$ Efforts have been made to identify the interaction of protein molecules with the mesoporous metal oxides surface. ${ }^{22}$ Some of the important factors associated with protein conjugation are the mesopore structured diameter, surface-to-volume ratio, morphology, surface modification, ionic strength, isoelectric point and protein dimension. Surface chemistry on a metal oxide can promote better conjugation of protein molecules by the cre- ation of functional groups. ${ }^{23,24}$ Mesoporous 1D nanofibers are known to facilitate the attachment of protein biomarkers (antibodies) or DNA molecules due to their fascinating geometries that can provide numerous absorption sites on the nanofiber surface. ${ }^{25}$ For the conjugation of biomolecules, the physical absorption driven by electrostatic attraction is a direct approach for the attachment of biomolecules due to the higher isoelectric point (10.3) of ZnOnF allowing it to easily bind with the low isoelectric point protein molecules $(\sim 1-5) .{ }^{20}$ Although non-specific binding due to electrostatic interactions with foreign molecules may prove to be a disadvantage, this method has been widely explored. However, covalent interactions can overcome this limitation due to strong amide bond formation. The zinc oxide surface can be modified by surface treatments such as oxygen plasma, wet chemical, surface graft polymerization, co-electrospinning, etc. ${ }^{14}$ Carbon doped in $\mathrm{ZnO}$ not only helps to create surface functional groups but also improves the electrochemical performance of a biosensor. ${ }^{12,26}$ In addition, surface functional groups on the $\mathrm{ZnOnF}$ surface in covalent interactions may amplify the electrochemical impedance signal. Xu et al. fabricated a 1D channel of ordered mesoporous metal oxides to assemble functional proteins for electrochemical detection of hydrogen peroxide. ${ }^{27}$ Mondal et al. fabricated mesoporous metal-oxide nanofibers for the functionalization of cholesterol esterase and cholesterol oxidase enzyme molecules. ${ }^{12}$ Hong et al. synthesized $\mathrm{ZnO}$ nanowires for fluorescent cancer cell imaging. ${ }^{28}$ Ahmed et al. fabricated a glucose biosensor using ZnOnF and obtained a sensitivity $\left(70.2 \mu \mathrm{A} \mathrm{cm}{ }^{-2} \mathrm{mM}^{-1}\right)$ for glucose detection. ${ }^{13}$ Other nanostructured materials including carbon nanotubes, ${ }^{29}$ reduced graphene oxide- $\mathrm{SnO}_{2}$ nanocomposite, ${ }^{30}$ hydrazine coated gold nanoparticles, ${ }^{30}$ etc., have been used to detect cancer biomarkers. However, their limitations, such as poor sensitivity and limit of detection, can be improved by using porous morphology ZnOnFs. The mesoporous $1 \mathrm{D} \mathrm{ZnOnF}$ has not been explored for the fabrication of POC devices for cancer biomarker detection to date.

Here we explore oxygen plasma treated and untreated electrospun mesoporous zinc oxide nanofiber (ZnOnF) platforms for conjugation with the antibody of human epidermal growth factor receptor 2 (anti-ErbB2) and its use for the labelfree detection of ErbB2 via antigen-antibody interactions. Precursor materials such as a carrier polymer (polyacrylonitrile; PAN) and zinc acetate were utilized for the synthesis of $\mathrm{ZnOnF}$ mats via electrospinning followed by calcination. Shape, size and diameter of the nanofibers was controlled by varying the concentration of the precursor materials, solution viscosity, flow rate, applied electric field, etc. A small amount of residual carbon in the ZnOnFs was optimized for creating functional groups to get the best sensor performance by varying the calcination temperature. ZnOnFs deposited on indium tin oxide glass are conjugated with anti-ErbB2 via covalent as well as physical electrostatic interactions. Owing to the presence of residual carbon in ZnOnFs, the oxygen plasma treatment generated functional groups such as $-\mathrm{COOH}$, which make a strong amide bond $(\mathrm{C}-\mathrm{N})$ with protein molecules. The functional 
property of ZnOnFs and their shape, size, crystalline structure, etc., have been investigated using structural and morphological studies. Label-free detection of ErbB2 has been carried out using a protein conjugated ZnOnFs immunoelectrode via the electrochemical impedance spectroscopic technique.

\section{Results and discussion}

\section{Optimized synthesis and morphological studies of ZnOnFs}

The synthesis of electrospun $\mathrm{ZnO}$ nanofibers was accomplished using a chemical method at a temperature of about $60{ }^{\circ} \mathrm{C}$, including mixing of a metal acetate dihydrate salt, zinc acetate dihydrate (ZnA) with a polar basic aprotic solvent (DMF) followed by a high temperature $\left(500^{\circ} \mathrm{C}\right)$ thermal treatment. The use of DMF as a useful reaction media for the arrested basic hydrolysis of zinc salts has been reported earlier. ${ }^{31}$ In this approach, DMF controls the hydrolysis of zinc acetate dihydrate salts. ${ }^{32}$ The carrier polymer (PAN) dissolved in DMF acts as a template and controls the growth kinetics of ZnO. This precursor undergoes hydrolysis in the presence of moisture and forms a $\mathrm{ZnO}$ gel in the electrospun nanofibers. The as-spun nanofiber mats were left in air for stabilization to permit the complete hydrolysis of $\mathrm{ZnA}$. PAN was removed by calcination leaving behind a continuous mesoporous $\mathrm{ZnO}$ nanofiber mat with some residual carbon content.

The vital parameters for the electrospinning of PAN-ZnA were optimized by Singh et al. ${ }^{33}$ to obtain uniform polymeric fibers with diameters in the range of $180-350 \mathrm{~nm}$. The role of the sacrificial polymer PAN in the electrospun solution was to increase the viscosity and therefore to control the viscoelastic behavior of the blend. The diameter of the electrospun nanofibers could be varied, which is a determining factor in the conductivity of the fibers and thereby increases biosensing efficiency. The lower fiber diameter leads to a higher specific surface area, therefore ensuring more reaction sites for enhancing the electrocatalytic efficacy. In order to optimize the diameter of the fibers, we have fixed the applied voltage, distance between the collector and the needle tip, ambient properties like temperature and humidity while we varied the electrospinning process parameters like polymer wt $\%$ and flow rate of the solution.

Electrospinning of $\mathrm{ZnO}$ occurs because of the high DC potential applied between the needle tip and the grounded rotating electrode. Because of the high voltage, the polymer drop at the needle tip starts to elongate and a thin polymer jet is ejected when the applied electric field strength beats the surface tension of the polymer solution. Stretching of this viscoelastic polymeric filament under the electric field coupled with the rapid solvent evaporation produces nanofibers deposited on the grounded rotating electrode. A rotating drum collector was used here to change the alignment of the collected fibers. At low rpm $(<500 \mathrm{rpm})$ the fibers were randomly deposited onto the collector surface. As the rotational speed of the drum was increased (usually $>1000 \mathrm{rpm}$ ) fibers were collected on the drum surface in a partially aligned orientation.
The diameter and morphology of the as-spun ZnOnFs were confirmed by the field emission-scanning electron microscopy (FE-SEM) studies. It was observed that at the solution flow rate of $75 \mu \mathrm{L} \mathrm{min}{ }^{-1}$ the diameter of the PAN-ZnA fibers decreases with the reduction in polymer concentration. The fibrous morphology is still retained up to a lower critical polymer concentration $\sim 2 \mathrm{wt} \%$. Below this concentration, electro-sprayed bead like structures were formed. A combination of beads and non-uniform fiber formation continues from 2 to $6 \mathrm{wt} \%$ as revealed by the FE-SEM micrograph (Fig. 1a). The formation of bead like structures occurs at low polymer wt\% which inhibits the Taylor cone formation. At low wt\% of PAN (low viscoelasticity of polymer solution) the liquid jet breakdowns easily and creates an electrospray of droplets/beads or beaded nonuniform PAN-ZnA fibers. A further increase in PAN concentration to $7.5 \mathrm{wt} \%$ produces a bead-free continuous fiber mat of increasing diameter (Fig. $1 \mathrm{~b}$ and c). At the very high polymer concentration of $14 \mathrm{wt} \%$ thick rough non-uniform diameter fibers (average diameter $\sim 0.85 \mu \mathrm{m}$ ) were obtained (Fig. 1d). Therefore, the $7.5 \mathrm{wt} \%$ PAN solution was found to be near optimal for producing bead-free, uniform diameter and nanofiber mats with fiber diameter around $270 \mathrm{~nm}$ (calculated from Fig. 1c).

When the flow rate of the PAN-ZnA solution (7.5 wt\%) was varied, the average fiber diameter was found to be $\sim 180 \mathrm{~nm}$

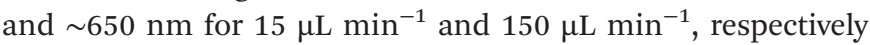
(Fig. 1e and f). In order to preserve the Taylor cone, the flow

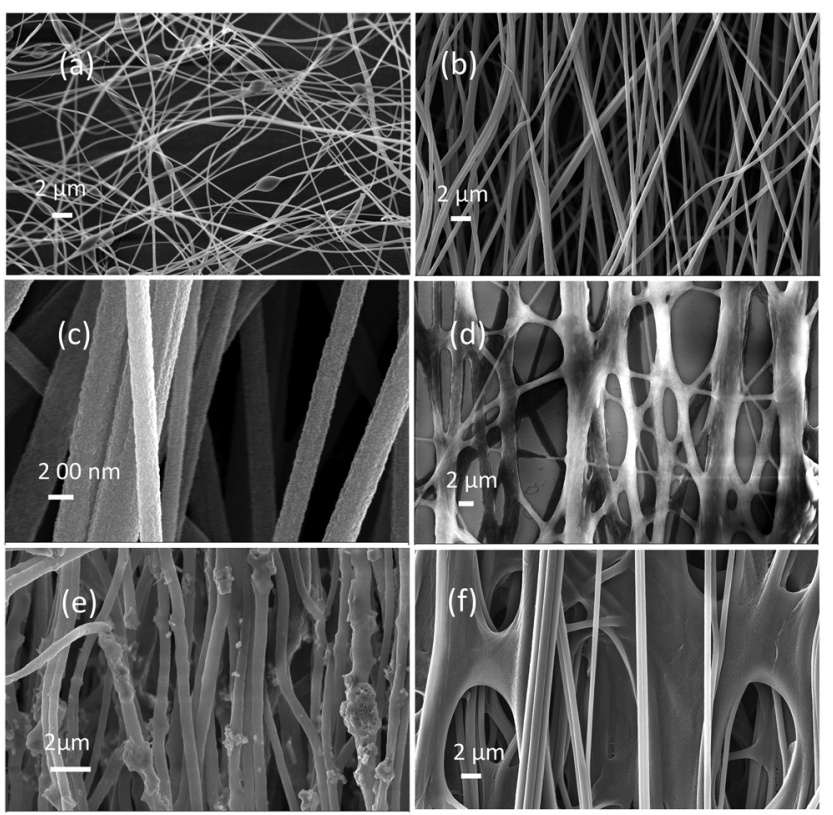

Fig. 1 FE-SEM micrographs for PAN-zinc acetate fibers electrospun at a $75 \mu \mathrm{L} \mathrm{min}^{-1}$ constant flow rate with different PAN concentrations, (a) beaded and non-uniform PAN-ZnA fibers observed at $3 \mathrm{wt} \%$, (b) more uniform in diameter bead-free fibers at $7.5 \mathrm{wt} \%$, (c) higher magnification image of (b) and (d) thicker fibers at $14 \mathrm{wt} \%$. (e-f) Morphology of a $7.5 \mathrm{wt} \%$ PAN-ZnA blend electrospun at different flow rates, (e) $15 \mu \mathrm{L}$ $\mathrm{min}^{-1}$ and (f) $150 \mu \mathrm{L} \mathrm{min}{ }^{-1}$. 
rate of the solution needs to be optimized accordingly. ${ }^{33}$ At very low flow rates $\left(15 \mu \mathrm{L} \mathrm{min}^{-1}\right)$ dry polymer at the tip of the needle was produced (Fig. 1e shows the beaded non uniform fibers), whereas a very high flow $\left(150 \mu \mathrm{L} \mathrm{min}^{-1}\right)$ produced large droplets and thick beaded fibers (Fig. 1f). Hence, it was found that a $7.5 \mathrm{wt} \%$ PAN-ZnA solution at a flow rate of $75 \mu \mathrm{L}$ $\mathrm{min}^{-1}$ and a $14 \mathrm{kV}$ DC voltage applied between the rotating electrode and needle tip placed $4 \mathrm{~cm}$ apart, produced near optimal thick nanofiber mats.

\section{Structural and electron microscopic studies}

Fig. 2 shows the morphology of the nanofibers after calcination at $500{ }^{\circ} \mathrm{C}$. Fig. 2a shows the low magnification SEM image of a free-standing $\mathrm{ZnOnF}$ mat (calcined at $500{ }^{\circ} \mathrm{C}$ ) consisting of partially aligned nanofibers. The as-spun nanofibers (Fig. 1b and c) are of smooth morphology, free from beads, uniform in diameter and are partially aligned in orientation. Calcination at $500{ }^{\circ} \mathrm{C}$ (Fig. $2 \mathrm{~b}$ and c) produced mesoporous continuous long nanofibers with an average diameter of about $80 \mathrm{~nm}$. Approximately a 70\% shrinkage in the fiber diameter was observed after calcination of the $7.5 \mathrm{wt} \%$ PAN-ZnA blend electrospun at a $75 \mu \mathrm{L} \mathrm{min}^{-1}$ flow rate. As calcination was optimized earlier, ${ }^{32}$ we calcined at $500{ }^{\circ} \mathrm{C}$ to retain a small amount

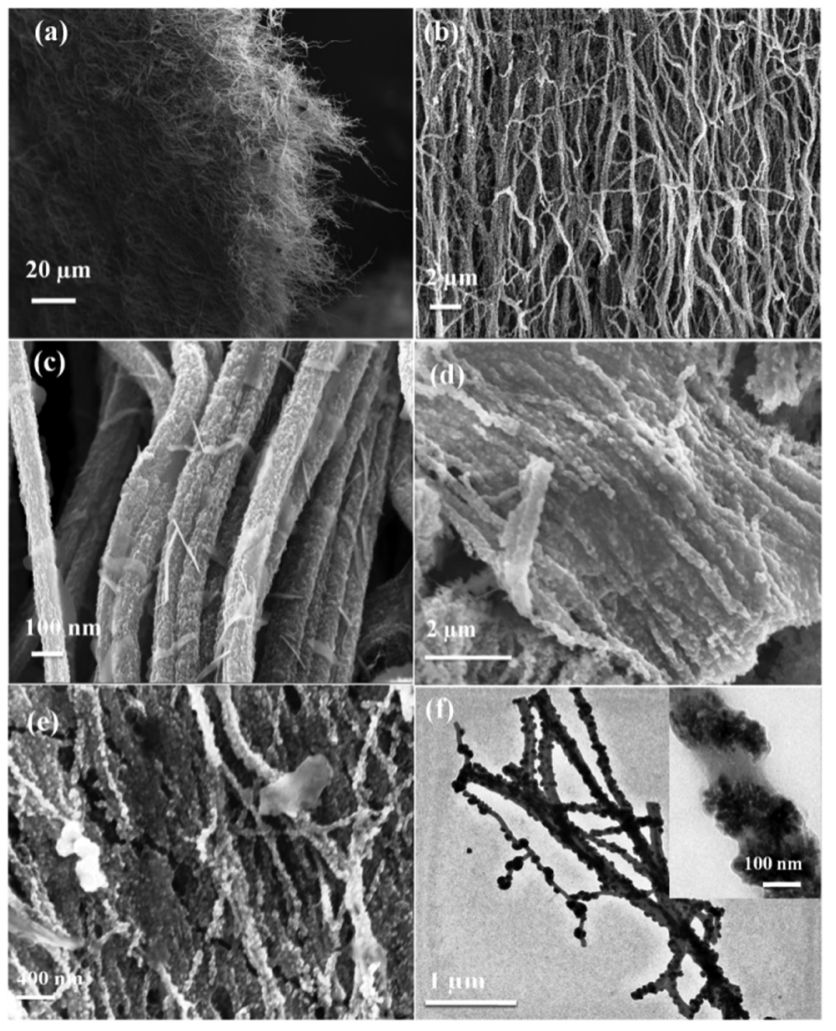

Fig. 2 FE-SEM images of $\mathrm{ZnOnF}$ produced at a calcination temperature of $500{ }^{\circ} \mathrm{C}$, (a) low magnification view of the calcined $\mathrm{ZnO}$ nanofiber free-standing mat, (b) higher magnification view of the $\mathrm{ZnO}$ mat and (c) individual high resolution image of $\mathrm{ZnOnFs}$. (d) FE-SEM for ErbB2 antibody loading on ZnOnF surface and (e) its higher magnification. TEM micrograph of $\mathrm{ZnOnFs}$ (f), inset shows an individual $\mathrm{ZnOnF}$. of residual carbon within the ZnOnF mats which may play an important role in protein conjugation after oxy-plasma treatment. Fig. 2d and e describe the morphology of the ZnOnFs after the loading of antibodies (anti-ErbB2). It is confirmed that ZnOnFs retain their fibrous morphology even after electrophoretic deposition (Fig. 2d and e). After immobilization via surface treatment, the SEM image of the anti-ErbB2 covered ZnOnF surface is shown in Fig. 2d. It can be seen that the plasma treated fibers show less propensity for aggregation. In addition, the mesopore structure of the ZnOnFs may enhance the absorption of protein molecules. The high resolution FE-SEM image after loading of the anti-ErbB2 molecules is shown in Fig. 2e.

Elementary analysis for $\mathrm{ZnOnF}$ and fZnOnF films and with antibody functionalization has been carried out to confirm the presence of elements in the prepared samples. Fig. S1(i, ESI $\dagger$ ) shows the energy dispersive X-ray (EDX) analysis spectra (embedded with the quantitative data) indicating removal of the polymer with carbon present after calcination at $500{ }^{\circ} \mathrm{C}$ for $4 \mathrm{~h}$ (a). After antibody functionalization, an EDX peak appears for the carbon element (atomic ratio; $12.2 \%$ ) indicating the presence of antibody molecules on the ZnOnF surface. Fig. S1 (ii, ESI $\dagger$ ) shows the EDX analysis spectra (embedded with the quantitative data) indicating removal of the polymer with small amounts of residual carbon present after calcination. After protein conjugation in the EDX spectra, elements such as phosphorus $(\mathrm{P})$, calcium $(\mathrm{Ca})$, potassium $(\mathrm{K})$ and chlorine $(\mathrm{Cl})$ found in the sample may be due to PBS with antibody on the fZnOnF transducer surface (Fig. S1(ii), ESI $\dagger$ ). However, the atomic ratio of carbon elements is found to increase indicating biofunctionalization of antibody on the fZnOnF transducer.

The microscopic structure of electrospun ZnOnFs obtained after calcination was investigated by TEM studies. The diameter of calcined ZnOnFs is around 60-120 nm after calcination at $500{ }^{\circ} \mathrm{C}$. Fig. $2 \mathrm{f}$ shows the TEM images of $\mathrm{ZnO}$ fibers. It is easy to identify $\mathrm{ZnO}$ grains from the magnified image shown in the inset of Fig. 2f. This suggests that ZnOnFs crystallized sufficiently due to the high calcination temperature. The average crystallite size of $\mathrm{ZnO}$ within the nanofibers is found to be $\sim 32 \mathrm{~nm}$ by analyzing TEM micrographs (analyzed by ImageJ software). The diameter of the fibers shrinks down to about $80 \mathrm{~nm}$ by heat treatment. The grains of $\mathrm{ZnO}$ are produced from ZnA salt and grow by oxidation at high calcination temperatures. The growth of the crystallites is along the fiber direction. It can be seen that the $\mathrm{ZnO}$ nanofibers contain small nanoparticles of $\mathrm{ZnO}$ along the fiber direction, however, the inset shows nanostructured flower like $\mathrm{ZnO}$ wrapped along the (101) direction of the ZnOnF, as confirmed by X-ray diffraction (XRD) studies.

The XRD studies were conducted to investigate the crystalline structure of synthesized ZnOnFs at a slow scan rate of $1^{\circ}$ $\min ^{-1}$. The presence of various peaks in the XRD pattern (Fig. 3i) indicates the wurtzite structure of crystalline zinc oxide with the peaks matched to the JCPDS card no. 36-1451 (lattice parameters: $a=3.249 \AA$ and $c=5.206 \AA$ ). The crystallite size of zinc oxide (calcined at $500{ }^{\circ} \mathrm{C}$ ) was calculated as 

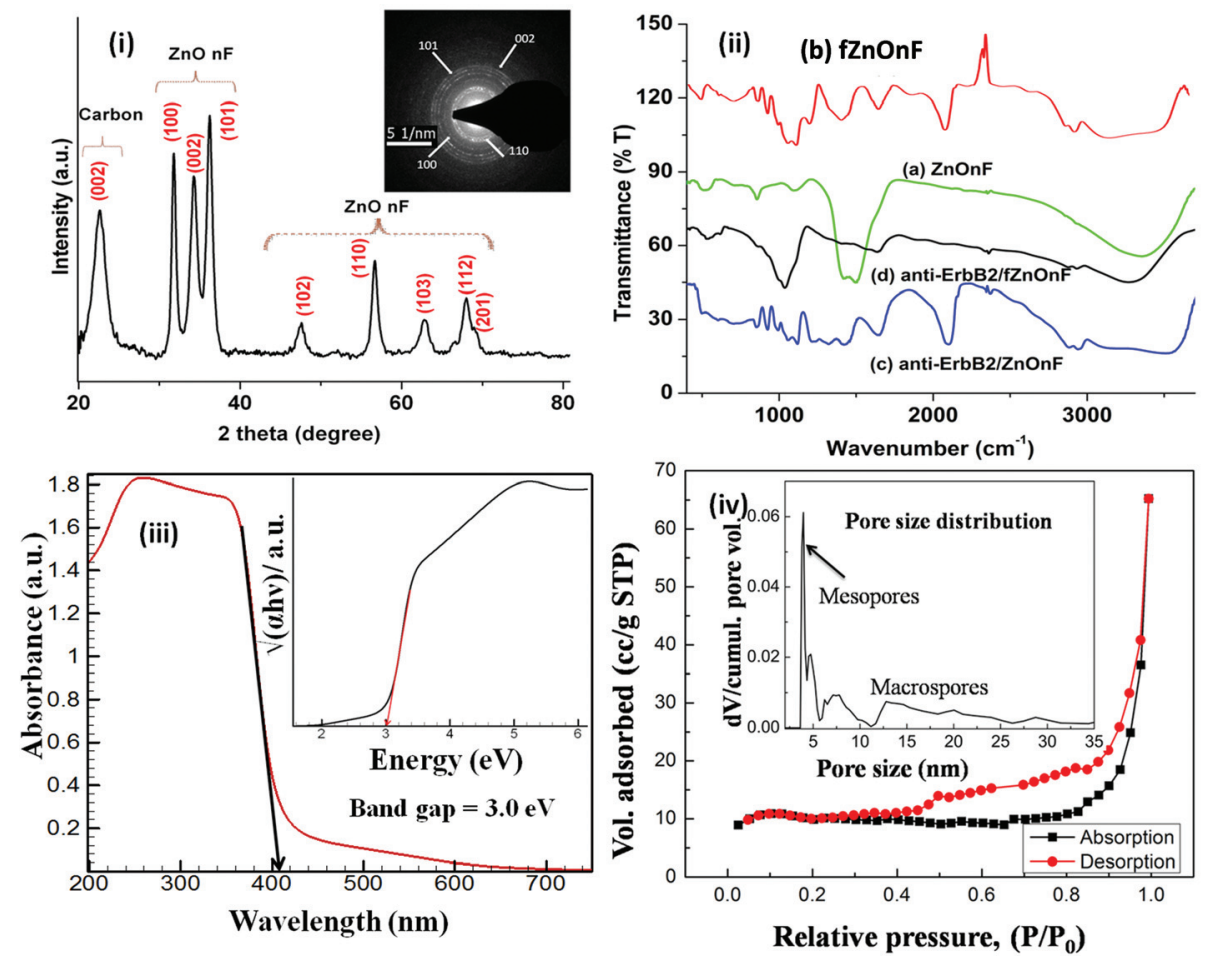

Fig. 3 (i) X-ray diffraction of synthesized zinc oxide nanofibers calcined at $500{ }^{\circ} \mathrm{C}$, inset shows the electron diffraction pattern of ZnOnFs, (ii) $\mathrm{FT}$-IR spectra of various fabricated electrodes, (iii) absorption spectra of ZnOnFs, (inset: Tauc plot for the band gap calculation), (iv) BET data for ZnOnFs (inset: pore size distribution).

$35.35 \mathrm{~nm}$ for the (101) plane using the Debye-Scherrer equation. This indicates that the zinc oxide crystallites grow along the (101) direction. A peak was found for the (002) reflection plane at $2 \theta=22.6^{\circ}$ due to the residual carbon content. In the UV-visible spectra, it can be seen that absorption increases from $420 \mathrm{~nm}$ to $250 \mathrm{~nm}$ and the maximum is at $350 \mathrm{~nm}$ due to the quantum confinement effect indicating the formation of nanostructured zinc oxide (Fig. 3iii). The estimated band gap $(3.0 \mathrm{eV})$ of ZnOnFs is reduced compared to bulk zinc oxide. Reduction of the band gap for the synthesized zinc oxide nanofibers is due to the blue shift which may help in enhancing electron conduction during electrochemical measurements.

To investigate the porosity of synthesized ZnOnFs, we conducted the Brunauer-Emmett-Teller (BET) measurement. For sampling the specific surface area, the sample was degassed in a vacuum at $200{ }^{\circ} \mathrm{C}$ for $8 \mathrm{~h}$ and then the nitrogen adsorption and desorption isotherms were recorded (Fig. 3iv). The pore size distribution was analyzed using non-local density functional theory from the adsorption branch of the isotherm (Fig. 3iv, inset). The volume was calculated from the adsorbed nitrogen gas at a relative pressure $\left(P / P_{\mathrm{o}}\right)$ of 0.9994 . The total BET surface area was found to be $\sim 28.07 \mathrm{~m}^{2} \mathrm{~g}^{-1}$ with an average pore diameter of about $14.41 \mathrm{~nm}$ and all the pores were below $326.9 \mathrm{~nm}$ in size. The pore volume was estimated by the BJH desorption method as $10.11 \times 10^{-2} \mathrm{cc} \mathrm{g}^{-1}$, where the micro, meso and macropore volumes were distributed around $0.0115,0.057$ and $0.03 \mathrm{~cm}^{3} \mathrm{~g}^{-1}$, respectively. Thus, the results indicate that ZnOnFs consist of meso (57\%) and macropores $(31.7 \%)$ with a fraction of around $11 \%$ micropores. The mesoporous structure of zinc oxide fibers may enhance the loading capacity of protein cancer biomarkers.

To confirm the available functional groups and formation of chemical bonding on the ZnOnF surface, FT-IR studies were performed (Fig. 3ii). In the finger print region of the spectra, the peak seen at $533 \mathrm{~cm}^{-1}$ is due to the $\mathrm{Zn}-\mathrm{O}$ vibration bend in the sample. A wide absorption peak (curve a) was observed at $3357 \mathrm{~cm}^{-1}$ corresponding to the $\mathrm{Zn}-\mathrm{OH}$ stretching in the ZnOnF sample. The strong absorption peak found at $1100 \mathrm{~cm}^{-1}$ reveals the presence of the $\mathrm{O}-\mathrm{C}$ group on the surface of ZnOnFs. The peak at $850 \mathrm{~cm}^{-1}$ indicates $\mathrm{C}-\mathrm{H}$ bending in the finger print region of the sample. The characteristic peak at $1422 \mathrm{~cm}^{-1}$ is assigned to $\mathrm{C}-\mathrm{O}-\mathrm{H}$ bending while at $1500 \mathrm{~cm}^{-1}$ is assigned to $\mathrm{C}=\mathrm{C}$ bending. However, oxygen plasma treatment of the $\mathrm{ZnOnF}$ surface changed the peak position toward higher wavenumbers, from $1100 \mathrm{~cm}^{-1}$ to $1130 \mathrm{~cm}^{-1}$, and peak intensities were increased (curve b). A peak observed at $1649 \mathrm{~cm}^{-1}$ reveals the presence of the carboxyl group on fZnOnFs due to oxygen plasma treatment indicating a $-\mathrm{COOH}$ functionalized surface (Mondal et al.). ${ }^{12}$ The additional peak found at $2070 \mathrm{~cm}^{-1}$ is due to the absorption of $\mathrm{CO}_{2}$ on the fZnOnF surface. After antibody (anti-ErbB2) functionalization on the fZnOnF surface, the peaks as seen in spectra $\mathrm{c}$ and $\mathrm{d}$ are found to disappear, indicating the absorp- 
tion of the antibody on the fZnOnF surface. A broad absorption peak found at $1040 \mathrm{~cm}^{-1}$ (curve d) due to $\mathrm{C}-\mathrm{N}$ bending arises from the covalent interaction of $-\mathrm{COOH}$ groups and $-\mathrm{NH}_{2}$ groups of the fZnOnFs and anti-ErbB2, respectively. In both spectra, a peak at $1631 \mathrm{~cm}^{-1}$, which is due to the $\mathrm{N}-\mathrm{H}$ stretching of the amide II bond, results after antibody ErbB2 absorption on ZnOnFs (c) and fZnOnFs (d).

\section{Electrochemical properties}

Cyclic voltammetric studies were conducted to investigate the electrochemical properties for mesoporous $\mathrm{ZnOnF}$ based electrodes in a wide potential range $(-0.6$ to $0.7 \mathrm{~V})$. Curves show excellent oxidation and reduction peaks for the fabricated electrodes in the presence of the $\left[\mathrm{Fe}(\mathrm{CN})_{6}\right]^{3-/ 4-}$ mediator (Fig. 4i). The ZnOnF-ITO electrode shows an oxidation peak potential of $0.37 \mathrm{~V}$ and current at $426 \mu \mathrm{A}$. Surface oxy-plasma treatment on the $\mathrm{ZnOnF}$ surface results in a decreased oxidation current due to the creation of functional groups $(-\mathrm{COOH},-\mathrm{OH}$ etc.). The peak-to-peak separation voltage decreases to $0.32 \mathrm{~V}$ for the fZnOnF-ITO electrode compared to the ZnOnF-ITO at 0.45 V. Anti-ErbB2 and BSA immobilization on the ZnOnF electrode via electrostatic interactions obstructs $\left[\mathrm{Fe}(\mathrm{CN})_{6}\right]^{3-/ 4-}$ diffusion of electrons from the bulk solution resulting in a decreased current $(283 \mu \mathrm{A})$. Again, it is observed that the immobilization of Anti-ErbB2 and BSA via covalent interactions followed by oxygen plasma treatment (anti-ErbB2-fZnOnF-ITO) reduces the electrochemical current compared to fZnOnF-ITO electrode $(266 \mu \mathrm{A})$. Conjugation of these protein molecules on the ZnOnF surface perhaps acts as a barrier to electron transportation from the bulk solution towards the electrode resulting in a decreased electrochemical current. Table $\mathrm{S} 1 \dagger$ shows the diffusion co-efficients of various fabricated electrodes. It has been observed that the higher diffusion co-efficient $(8.23 \times$ $10^{-8} \mathrm{~cm}^{2} \mathrm{~s}^{-1}$ ) of the porous morphology ZnOnF electrode may be due to the high aspect ratio of mesoporous ZnOnFs that provides an electron conduction path for transportation from the bulk solution resulting in the higher diffusion of ions towards the current collector. The diffusion of electrons due to redox conversation for porous $\mathrm{fZnOnF}$ electrodes has been estimated as $6.28 \times 10^{-8} \mathrm{~cm}^{2} \mathrm{~s}^{-1}$. The functionalized $\mathrm{ZnO}$ nanofibers base electrode contains $-\mathrm{COOH}$ groups on its surface that may obstruct electron diffusion from the bulk solution compared to ZnOnFs without functionalization. However, antibody functionalization on ZnOnFs decreases the diffusion of electrons $\left(3.64 \times 10^{-8} \mathrm{~cm}^{2} \mathrm{~s}^{-1}\right)$ due to the attachment of insulating antibody molecules on their surface. The antibody functionalized electrode using fZnOnF again shows a decreased diffusion of electrons $\left(3.13 \times 10^{-8} \mathrm{~cm}^{2} \mathrm{~s}^{-1}\right)$, which may be due to covalent interactions indicating that a higher amount of antibody molecules can be attached to fZnOnFs. In scan rate studies, we have measured the $\mathrm{CV}$ response of both fabricated bioelectrodes (anti-ErbB2-fZnOnFs and anti-ErbB2-ZnOnFs)
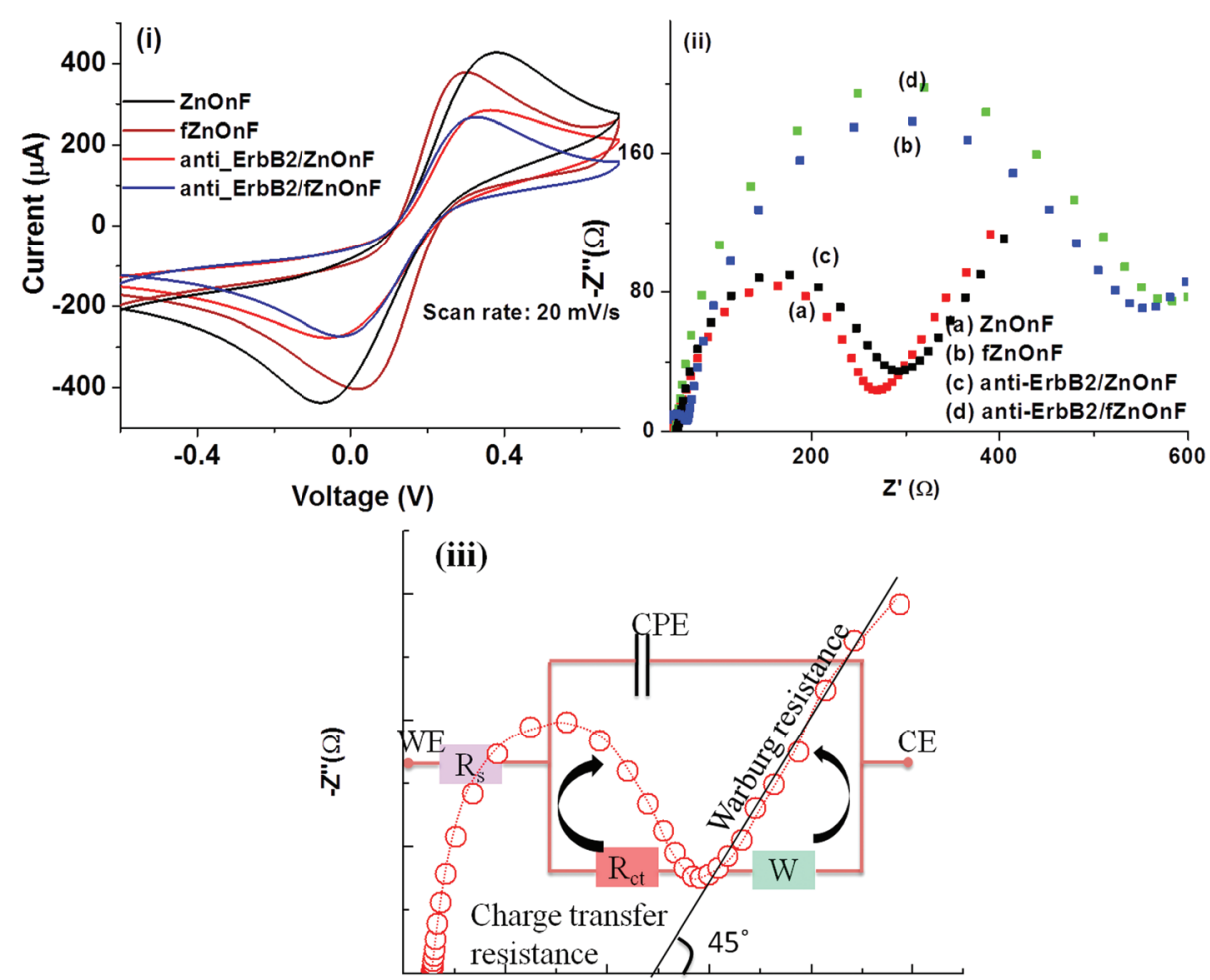

$\mathbf{Z}^{\prime}(\Omega)$

Fig. 4 (i) Cyclic voltammetric studies for various electrodes and immunoelectrodes at $20 \mathrm{mV} \mathrm{s}^{-1}$, (ii) electrochemical impedance analysis for the fabricated electrodes and (iii) equivalent circuit model for a typical Nyquist plot for impedance studies. 
as a function of scan rate $\left(20-120 \mathrm{mV} \mathrm{s}^{-1}\right)$ (Fig. S2, ESI $\dagger$ ). The peak-to-peak separation voltage of both bioelectrodes increases and shifts toward the higher potential side with increasing scan rates. The anodic and cathodic peak currents of both bioelectrodes are found to be proportional and inversely proportional with the square root of the scan rate. Thus, the results of these studies indicate that both bioelectrodes show a surface controlled process. ${ }^{34}$ The variation of the peak (anodic and cathodic) current for both bioelectrodes as a function of the square root of the scan rate follows eqn (1)-(4):

$$
\begin{aligned}
I_{\mathrm{pa}}(\text { anti-ErbB2-ZnOnFs })= & 55 \mu\left[\mathrm{A}^{2} \mathrm{mV}^{-1} \mathrm{~s}\right]^{1 / 2} \\
& \times \text { Scan rate }\left[\mathrm{mV} \mathrm{s}^{-1}\right]^{1 / 2}+91 \mu \mathrm{A}
\end{aligned}
$$

$$
\begin{aligned}
I_{\mathrm{pc}}(\operatorname{anti}-E r b B 2-Z n O n F s)= & -39 \mu\left[\mathrm{A}^{2} \mathrm{mV}^{-1} \mathrm{~s}\right]^{1 / 2} \\
& \times \text { Scan rate }\left[\mathrm{mV} \mathrm{s}^{-1}\right]^{1 / 2}-151 \mu \mathrm{A}
\end{aligned}
$$

$$
\begin{aligned}
I_{\mathrm{pa}}(\text { anti-ErbB2-fZnOnFs })= & 43 \mu\left[\mathrm{A}^{2} \mathrm{mV}^{-1} \mathrm{~s}\right]^{1 / 2} \\
& \times \text { Scan rate }\left[\mathrm{mV} \mathrm{s}^{-1}\right]^{1 / 2}+83 \mu \mathrm{A}
\end{aligned}
$$

$$
\begin{aligned}
I_{\mathrm{pc}}(\text { anti-ErbB2-fZnOnFs })= & -27 \mu\left[\mathrm{A}^{2} \mathrm{mV}^{-1} \mathrm{~s}\right]^{1 / 2} \\
& \times \text { Scan rate }\left[\mathrm{mV} \mathrm{s}^{-1}\right]^{1 / 2}-158 \mu \mathrm{A}
\end{aligned}
$$

To investigate the interfacial property of the electrode-electrolyte, we carried out electrochemical impedance spectroscopy (EIS) measurements as a function of frequency (0.01 $10^{5} \mathrm{~Hz}$ ). The EIS spectra obtained for the fabricated electrodes are shown in Fig. 4ii. The charge transfer resistance $\left(R_{\mathrm{ct}}\right)$ and constant phase element (CPE) of various electrodes has been obtained from Nyquist plots (equivalent circuit diagram shown in Fig. 4iii) using EIS measurements (Table S1†). The presence of functional groups on the fZnOnF surface increases the $R_{\mathrm{ct}}$ value, however, the CPE value is decreased compared to the fZnOnF electrode. This may be due to the enhanced hydrophilic nature of ZnOnFs after surface oxygen plasma treatment. Protein (antibody and BSA) conjugation on the ZnOnF surface further enhances the $R_{\text {ct }}$ value. In addition, oxygen plasma treatment of the $\mathrm{ZnOnF}$ electrode improves the loading of protein molecules, which hinders the diffusion of electrons produced in the oxidation/reduction of $\left[\mathrm{Fe}(\mathrm{CN})_{6}\right]^{3-/ 4-}$ ions resulting in a higher $R_{\mathrm{ct}}(451 \Omega)$ compared to the physical absorption of protein molecules on the ZnOnF electrode surface $\left(R_{\mathrm{ct}}=412 \Omega\right)$. The reduction of the constant phase element after protein immobilization indicates an attachment of biomolecules on the ZnOnF surface resulting in an increased electric double layer formed between the conductive ITO electrode and an adjacent electrolyte. Interestingly, the capacitive behavior of the $\mathrm{ZnOnF}$ electrode is changed by the incorporation of functional groups on its surface for protein conjugations. The low value of CPE for the anti-ErbB2-ZnOnF immunoelectrode $\left(2.31 \times 10^{-5} \mathrm{~F}\right)$ indicates a higher double layer distance compared to the anti-ErbB2-fZnOnF immunoelectrode $\left(2.72 \times 10^{-5} \mathrm{~F}\right)$.

The heterogeneous electron transfer rate constant $\left(k_{0}\right)$ in the presence of the $\left[\mathrm{Fe}(\mathrm{CN})_{6}\right]^{3-/ 4-}$ mediator has been determined for various fabricated electrodes, as shown in Table S1. $\uparrow$ The value of $k_{0}$ can be estimated using the relation $k_{0}=R T / n^{2} F^{2} A R_{\mathrm{ct}} C$, where $n$ is the electron transferring constant of the redox couple, $F$ is the Faraday constant, $A$ is the area of the electrode, $R$ is the gas constant, $T$ is the temperature, $R_{\mathrm{ct}}$ is the charge transfer resistance and $C$ is the concentration of the redox mediator. It is observed from Table $\mathrm{S} 1 \uparrow$ that the highest electron transfer rate $\left(11.1 \times 10^{-7} \mathrm{~cm} \mathrm{~s}^{-1}\right)$ occurs for the pristine zinc oxide electrode, but declines for the oxy-plasma treated and antibody functionalized electrodes with or without plasma treatment. Surface treatment of the $\mathrm{ZnOnF}$ electrode at the electrode-electrolyte interface could be responsible for the observed dependence of the electron-transfer kinetics of the $\left[\mathrm{Fe}(\mathrm{CN})_{6}\right]^{3-/ 4-}$ mediator. A higher impedance or slower charge transfer rate of the anti-ErbB2-fZnOnF immunoelectrode (47.3 $\times 10^{-7} \mathrm{~cm} \mathrm{~s}^{-1}$ ) results in the higher capacitance creation at the electrode surface. The surface coverage $(\theta)$ can be calculated using the relation $\theta=1-R_{\mathrm{ct}}$ (electrode) $/ R_{\mathrm{ct}}$ (bioelectrode), where $\theta$ is the fraction of the occupied binding sites, and $R_{\mathrm{ct}}$ (electrode) and $R_{\mathrm{ct}}$ (bioelectrode) are the surface specific charge transfer resistances of the $\mathrm{ZnOnF}$ electrode and the anti-ErbB2-ZnOnF bioelectrode, respectively. We have estimated the surface concentration of physisorption and covalent attachment of the antibody on the ZnOnF electrode surface as $53.6 \%$ and $54.1 \%$, respectively. Thus a $0.5 \%$ enhancement of surface coverage of covalently immobilized antibodies occurs on the electrode surface via plasma treatment. The surface coverage of the electrode can enhance the electrochemical sensitivity and LOD of the biosensor device.

\section{Electrochemical detection of the cancer biomarker}

The electrochemical impedance spectroscopy (EIS) technique was utilized for the label-free detection of human epidermal growth factor receptor 2 (ErbB2). Anti-ErbB2 was functionalized on the $\mathrm{ZnOnF}$ electrode surface with and without the oxygen plasma treatment for the detection of various concentrations of ErbB2 (1.0 fM-0.5 $\mu \mathrm{M})$. Fig. 5i and ii show the EIS spectra of anti-ErbB2-ZnOnF and anti-ErbB2-fZnOnF electrodes as a function of ErbB-2 concentration. These biosensors can be regenerated by a glycine solution $(0.2 \mathrm{M})$ at $\mathrm{pH} 2.4$ for two minutes which re-generated $96.5 \%$ of the initial signal. It has been observed that the charge transfer resistance in these EIS spectra increases with increasing concentration of ErbB-2. Fig. 5iii and iv show the plots of the charge transfer resistances $\left(R_{\mathrm{ct}}\right)$ and logarithmic value of the ErbB-2 concentrations for anti-ErbB2-ZnOnF and anti-ErbB2-fZnOnF immunoelectrodes. Interaction of specific binding sites of antigen and antibody on the transducer surface form an immunocomplex layer that obstructs the electron transfer from the bulk solution resulting in a higher $R_{\mathrm{ct}}$. It was found that the $R_{\mathrm{ct}}$ value for the anti-ErbB2-fZnOnF electrode is higher compared to the anti-ErbB2-ZnOnF electrode (Fig. 5iii), which may be due to 

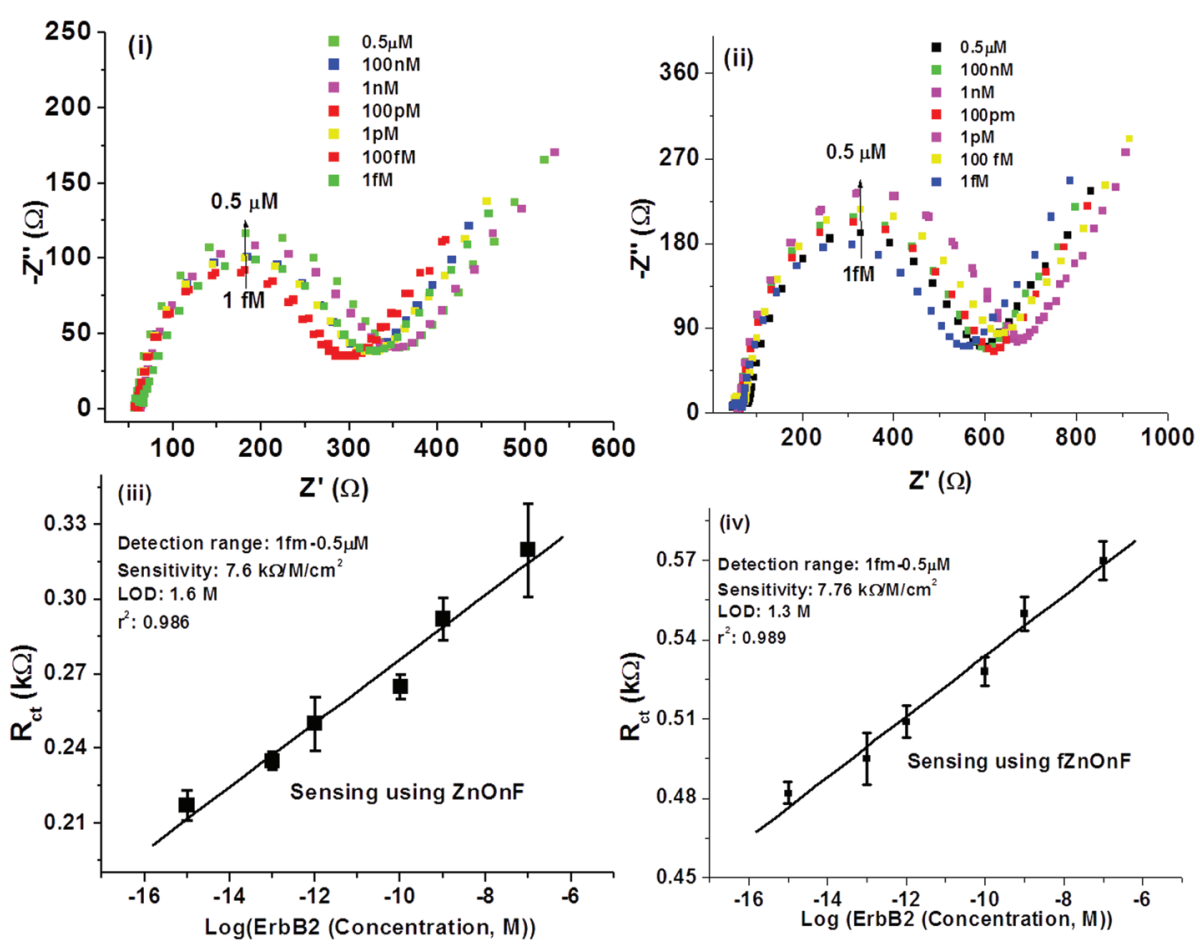

Fig. 5 Impedance spectra for the (i) anti-ErbB2-ZnOnF and (ii) anti-ErbB2-fZnOnF immunoelectrodes as a function of ErbB2 concentration (1.0 $\mathrm{fM}-0.5 \mu \mathrm{M})$ ]. Sensor calibration plots for the (iii) anti-ErbB2-ZnOnF and (iv) anti-ErbB2-fZnOnF immunoelectrodes using a logarithmic scale.

the available functional groups created after the oxygen plasma treatment on the $\mathrm{ZnOnF}$ surface. These fabricated immunoelectrodes can detect $1.0 \mathrm{fM}$ concentration of ErbB2 within a range of concentration $\left(1.0 \mathrm{fM}-0.5 \mu \mathrm{M}\right.$ or $4.34 \times 10^{-5}$ $\left.2.17 \times 10^{4} \mathrm{ng} \mathrm{mL}^{-1}\right)$. A comparable sensitivity for both antiErbB2-fZnOnF (7.76 $\mathrm{k} \Omega \mathrm{M}^{-1} \mathrm{~cm}^{-2}$ ) and anti-ErbB2-ZnOnF (7.6 $\mathrm{k} \Omega \mathrm{M}^{-1} \mathrm{~cm}^{-2}$ ) electrodes was obtained. The limit of detection (LOD) for anti-ErbB2-fZnOnF is low (1.3 M) compared to the anti-ErbB2-ZnOnF (1.6 M) immunoelectrode. The three dimensional structure of mesoporous ZnOnFs improves the loading of protein molecules such as anti-ErbB2 and BSA resulting in a higher sensitivity in both cases and an improved stability of the immunosensor. Myung et al. developed a 3D structure of a graphene based biosensor which significantly increases the surface-to-volume ratio, thereby improving the detection limits to $1.0 \mathrm{pM}$ for HER2 and $100 \mathrm{pM}$ for EGFR breast cancer biomarkers. ${ }^{30}$ Tehrani et al. fabricated microchannels of a chemically modified multi-layer epitaxial graphene based biosensor for the detection of a cancer biomarker (8-hydroxydeoxyguanosine) that is sensitive up to $0.35 \mathrm{nM}^{35}$ However, the prepared biosensors can detect lower concentrations of ErbB2 compared to those of the reported biosensors (Table 1), ${ }^{29,30,36,37}$ this may perhaps be because of the high surface area of mesoporous ZnOnFs for antibody conjugation. Table 1 shows the breast cancer sensing characteristics for the fabricated immunoelectrodes alongwith those reported in literature. The control experiments for the $\mathrm{ZnOnF}$ and $\mathrm{fZnOnF}$ electrodes were carried out with and without antibody (antiErbB2) immobilization (Fig. S3, ESI $\dagger$ ). The $R_{\text {ct }}$ values for the electrodes are slightly changed in the presence of ErbB2 and the values are not systematic. This may be due to the nonspecific binding with protein molecules in the presence of an antigen solution. The detection time was found to be low (128 s) for the oxy-plasma treated immunoelectrode (fZnOnFs) compared to the untreated $\mathrm{ZnOnF}$ immunoelectrode (141 s) as shown in Fig. S4 (ESI $\dagger$ ). Compared to the other reported biosensors for breast cancer detection, the biosensor fabricated here can detect a much lower concentration (1.0 fM) of the biomarker (Table 1$)$. The association constant $\left(K_{\mathrm{a}}\right)$ for both the fZnOnF and ZnOnF based immunoelectrodes is higher (404.8 $\mathrm{kM}^{-1} \mathrm{~s}^{-1}$ and $165.6 \mathrm{kM}^{-1} \mathrm{~s}^{-1}$, respectively) compared to other immunosensors reported. ${ }^{38,39}$ Thus the higher $K_{\mathrm{a}}$ of the fZnOnF electrode indicates a high affinity towards the ErbB2 antigen. This may perhaps be due to the covalent immobilization of the antibody compared to the ZnOnF immunoelectrode.

Reproducibility was investigated by detecting $100 \mathrm{nM}$ of ErbB2 with five different immunoelectrodes under similar conditions. The relative standard deviations (RSD) for the antiErbB2-ZnOnF and anti-ErbB2-fZnOnF immunoelectrodes were found to be $5.9 \%$ and $2.3 \%$, respectively, which suggest the good precision and reproducibility of the immunosensors. The stability of the anti-ErbB2-ZnOnFs and anti-ErbB2fZnOnFs was investigated for 50 days. The anti-ErbB2-fZnOnF immunosensor retained $95.5 \%$ of the initial signal value, while an $88 \%$ signal retention was observed after 50 days in the case of the anti-ErbB2-ZnOnF immunoelectrode (Fig. S5, $\mathrm{ESI} \dagger)$. To investigate the interference of the immunosensors, 
Table 1 Sensing performances of fabricated electrodes with the reported literature for the detection of breast cancer biomarker

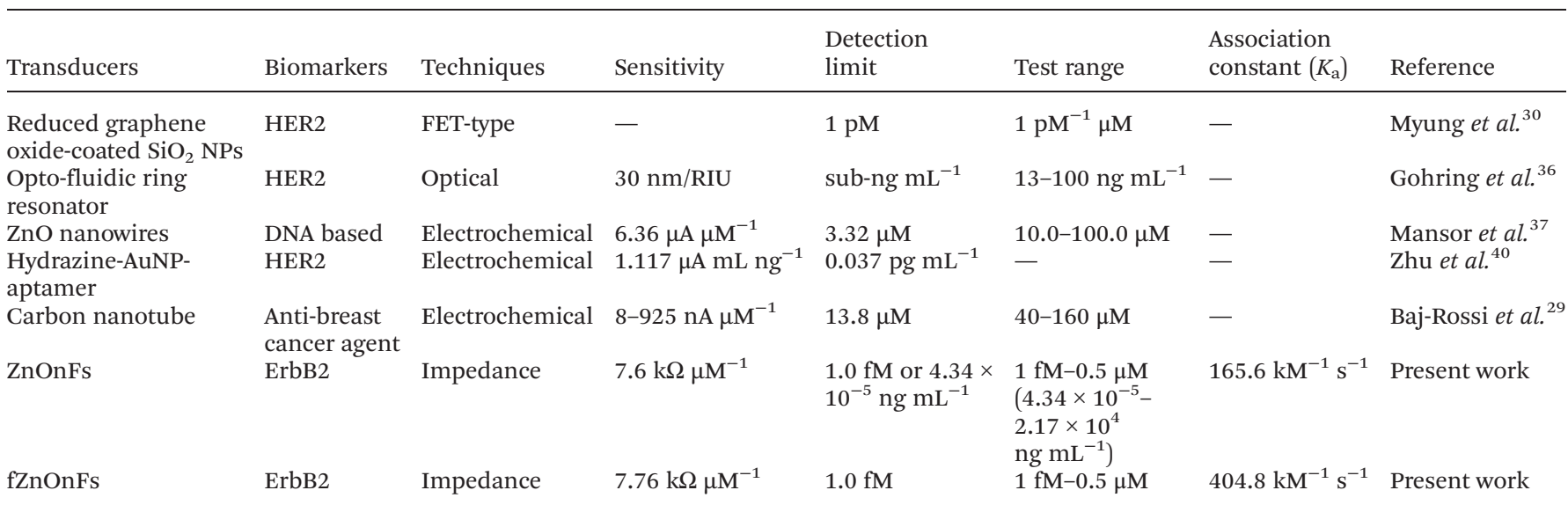

the responses towards human IgG, cholesterol, PSA, glucose and also with the BSA were determined considering their significance in serum. The immunosensors were tested in the presence of $100 \mathrm{nM}$ of ErbB2 with normal concentrations of interferents (Fig. S6, ESI $\dagger$ ). The variation of charge transfer resistance $\left(R_{\mathrm{ct}}\right)$ due to the interfering substances was found to be less than $5 \%$ for both immunoelectrodes, indicating good selectivity.

\section{Conclusions}

We have demonstrated the fabrication of an efficient, rapid detection and highly sensitive label-free immunosensor using antibody anchoring electrospun mesoporous zinc oxide nanofibers. The electrospun fibrous electrode was treated with oxygen plasma to enhance the loading capacity of protein (antibody) molecules via EDC-NHS covalent surface chemistry. Electrochemical detection by the protein functionalized fibrous immunoelectrode using the impedance spectroscopic method was carried out with and without oxy-plasma treatment on the ZnOnF surface for the concentration of the breast cancer biomarker. In these prepared immunosensors the freestanding mats of partially aligned ZnOnFs (diameters less than $100 \mathrm{~nm}$ ) were controlled during the synthesis of the nanofibers, however, after the electrophoretic deposition of the ZnOnFs onto the indium tin oxide surface their morphological order changed due to high the deposition potential. The electrochemical property of the ZnOnF based electrode changed with the protein molecules. The heterogeneous electron transfer constant and diffusion coefficient of the antibody conjugated ZnOnF electrode decreased, which influences the impedance signal during ErbB2 detection. Thus, both fabricated immunoelectrodes (oxy-plasma treated and untreated) show a comparable analytical performance for the detection of the breast cancer biomarker resulting in an insignificant change in sensitivity and a significant change in the heterogeneous electron transfer rate constant, detection time and association constant. These immunosensors provide a fast response of $128 \mathrm{~s}$ and $141 \mathrm{~s}$ for the $\mathrm{fZnOnF}$ and $\mathrm{ZnOnF}$ bioelectrodes, respectively, and are capable of detecting femtomolar concentrations of ErbB2 in the wide range of $1 \mathrm{fM}-0.5$ $\mu \mathrm{M}$ without using any labeling agents with good sensitivity, stability, reproducibility and selectivity. A mesoporous ZnOnF based label-free immunosensor thus provides a suitable platform for the early detection of breast cancer and may also find applications in the efficient detection of a host of other biomarkers.

\section{Materials and methods}

\section{Materials}

$N, N$-Dimethyl-formamide (DMF) solvent was purchased from Fischer Scientific, India. Zinc acetate $\left(\mathrm{Zn}\left(\mathrm{CH}_{3} \mathrm{COO}\right)_{2}, 2 \mathrm{H}_{2} \mathrm{O}\right.$, 99\%), polyacrylonitrile (PAN $\left(M_{\mathrm{W}}=150000\right)$ ), naphthalene and anthracene were procured from Sigma-Aldrich, USA. Bovine serum albumin (BSA), $N$-hydroxysuccinimide (NHS) and $N$-ethyl- $N$-(3-dimethylaminopropyl carbodiimide) (EDC) were purchased from Sigma-Aldrich (USA). For stock solutions of epidermal growth factor receptor, 2ErbB-2 (human recombinant) we used $0.1 \mathrm{M}$ acetate buffer at $\mathrm{pH} 4\left(0.5 \mathrm{mg} \mathrm{ml}^{-1}\right)$. To dilute the stock solutions of ErbB2 we used acetate buffer (0.1 M). The polyclonal antibody of ErbB2 soluble in phosphate buffered saline (PBS) containing 30\% glycerol, 1\% BSA and $0.02 \%$ thimerosal was purchased from BioVision, USA. Deionized distilled water was from a Millipore water purification system. The BSA ( $\left.2 \mathrm{mg} \mathrm{mL} \mathrm{mL}^{-1}\right)$ solution was prepared in $50 \mathrm{mM}$ PBS (pH 7.4) containing $150 \mathrm{mM} \mathrm{NaCl}$.

\section{Instrumentation}

The synthesized ZnOnFs were analysed by Fourier-transform infra-red spectroscopy (FT-IR; Perkin-Elmer, Model 2000) before and after oxygen plasma treatment. The morphological shape and size of the ZnOnFs in different conditions were observed by Field emission scanning electron microscopy (FE-SEM; Supra 400VP, Zeiss, Germany). Energy-dispersive X-ray spectroscopy (EDX; Oxford Instruments) analyses for the 
elemental composition of the ZnOnFs was carried out. The X-ray diffraction (XRD; X'Pert Pro, PAN Analytical, Netherlands, X-ray system with $\mathrm{Cu} \mathrm{K} \alpha$ radiation and $\lambda=1.54 \AA$ ) pattern confirmed the crystalline structure of the synthesized ZnOnFs. UVvisible analysis (Varian Cary 50Bio, UV-vis spectrophotometer) was conducted to investigate the structural properties of ZnOnFs. Brunauer-Emmett-Teller (BET; Quantachrome Instruments, USA) was carried out to evaluate the pore size distribution and total surface area of ZnOnFs. Cyclic voltammetry and electrochemical impedance spectroscopy were performed using an Autolab Potentiostat/Galvanostat (Electrochemical analyzer; AUT-84275) in PBS at $\mathrm{pH} 7.4$ containing $5 \mathrm{mM}$ of $\left[\mathrm{Fe}(\mathrm{CN})_{6}\right]^{3-/ 4-}$ as a mediator. Platinum (Pt) and $\mathrm{Ag}-\mathrm{AgCl}$ electrodes were used for counter and reference electrodes, respectively.

\section{Synthesis of mesoporous ZnOnFs}

Synthesis of mesoporous $\mathrm{ZnO}$ nanofibers was demonstrated in a previously reported paper. $^{33}$ A pictorial representation is shown in Scheme 1 with the synthesis protocol of zinc oxide nanofibers. In brief, a $7.5 \mathrm{wt} \%$ PAN solution was prepared in DMF solvent and magnetically stirred for $1 \mathrm{~h}$ at $60{ }^{\circ} \mathrm{C}$. Then, $0.25 \mathrm{~g}$ of zinc acetate $(\mathrm{ZnA})$ was mixed in the prepared PAN solution and stirred for $6 \mathrm{~h}$ at $50{ }^{\circ} \mathrm{C}$ to obtain a homogenous PAN-ZnA solution. This PAN-ZnA solution was transferred to a plastic hypodermic syringe (Dispovan, Hindustan Syringes Medical Devices Ltd, India) and fed through a syringe pump (Harvard Apparatus, Holliston, MA) at a $75 \mu \mathrm{L} \mathrm{min}{ }^{-1}$ flow rate. An electric field $\left(3.5 \mathrm{kV} \mathrm{cm}^{-1}\right)$ was applied by a DC power source (Gamma High Voltage, Inc., High Bridge, NJ) between the needle tip and the rotating collector. The collector was electrically grounded and rotated at $3000 \mathrm{rpm}$. This rotating collection module consists of a rotating drum unit with a translational linear motion emitter.

The non-woven fibers were collected for $\sim 5 \mathrm{~h}$ to make a thick mat $(0.1 \mathrm{~mm})$ on a rotating drum electrode which was covered with an aluminium foil. This free-standing mat like structure of nanofibers was then heated in air at $165{ }^{\circ} \mathrm{C}$ for $3 \mathrm{~h}$ to stabilize, which removes the solvent and moisture present in the collected as-spun fibers. The above method was repeated by varying parameters like the concentration of the PAN solution and the solution flow rate at a constant electric field. All the experiments were carried out in a controlled

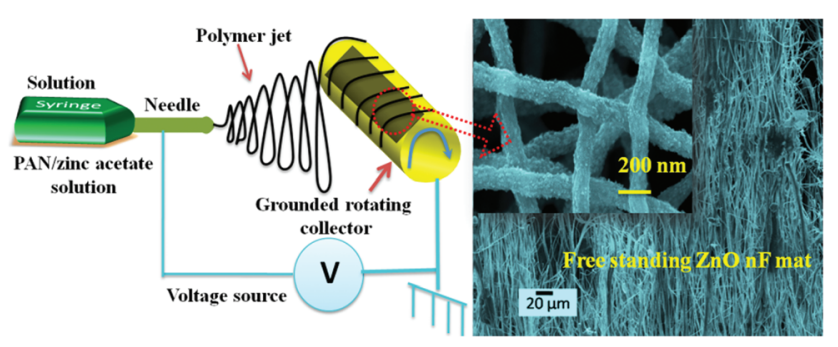

Scheme 1 Synthesis of electrospun ZnOnFs using a rotating drum collector. atmosphere at a room temperature of $25{ }^{\circ} \mathrm{C}, 32 \%$ humidity and at a $\mathrm{pH}$ value of 7 . The nanofiber mats thus formed were calcined at $500{ }^{\circ} \mathrm{C}\left(\right.$ ramp rate $\left.=5{ }^{\circ} \mathrm{C} \mathrm{min}^{-1}\right)$ for $2 \mathrm{~h}$.

\section{Electrode fabrication}

In order to fabricate the electrode, $3.5 \mathrm{mg}$ of an electrospun, partially aligned $\mathrm{ZnOnF}$ free-standing mat were added to $5 \mathrm{ml}$ of acetonitrile and sonicated (40 W, $0.25 \mathrm{~A}$ ) for $5 \mathrm{~h}$. During electrophoretic deposition, $1 \mathrm{~mL}$ of a $\mathrm{ZnOnF}$ suspended solution was added to $10 \mathrm{~mL}$ of an acetonitrile solution and sonicated. A platinum foil $(1 \mathrm{~cm} \times 2 \mathrm{~cm})$ and a pre-cleaned ITOcoated glass substrate (size; $0.5 \mathrm{~cm}^{2}$, sheet resistance; $30 \Omega$ $\mathrm{cm}^{-1}$ ) acted as the cathode and anode electrode, respectively. These two electrodes were positioned parallel to each other and separated by $1 \mathrm{~cm}$ and dipped in the $\mathrm{ZnOnF}$ colloidal suspension. A DC voltage of $60 \mathrm{~V}$ was applied between the two electrodes for $3 \mathrm{~min}$ in the presence of the $\mathrm{ZnOnF}$ colloidal suspension. The suspended ZnOnFs were deposited uniformly on the ITO electrode surface due to oppositely charged polarity of the applied potential and ZnOnFs. Then, the deposited films were removed from the ZnOnF suspension and washed several times with de-ionised water followed by drying at $100{ }^{\circ} \mathrm{C}$. Again, the $\mathrm{ZnOnF}$ electrode was treated with oxygen plasma for covalent functionalization (fZnOnF) with the antibody.

\section{Device fabrication for breast cancer diagnostics}

For antibody-ErbB2 functionalization, we explored both physical and covalent immobilizations. The anti-ErbB2-ZnOnF (without plasma treatment) and anti-ErbB2-fZnOnF (with plasma treatment) immunoelectrodes were used for the labelfree detection of epidermal growth factor receptor 2 (ErbB2; breast cancer biomarker) via antigen-antibody interactions. For physical immobilization the antibody-ErbB2 solution (0.02 $\mathrm{mg} \mathrm{mL}^{-1}$ ) was directly spread over the ZnOnF surface. The physical adsorption of anti-ErbB2 has been carried out without oxygen plasma treatment on the ZnOnF surface.

Prior to covalent functionalization, the $\mathrm{ZnOnF}$ based electrodes were oxygen plasma treated and the vacuum pressure was set to 0.005 mbar. The ZnOnF-ITO electrode with and without surface treatment was utilized for conjugation of the protein cancer biomarker (anti-ErbB2) via physical adsorption as well as covalent attachment. During plasma treatment, oxidation was performed in low intensity mode for $15 \mathrm{~min}$, oxidation voltage was kept at $450 \mathrm{~V}$ and pure oxygen was supplied. In this process, various residual organic impurities and weakly bound organic contaminations could be removed from the ZnOnF-ITO surface. Thus, the plasma treatment introduced various functional groups, such as $-\mathrm{COOH},-\mathrm{OH}$, etc., on ZnOnF-ITO and verified using FT-IR studies. The prepared electrodes were treated with EDC-NHS for $4 \mathrm{~h}$ to activate the functional groups $(-\mathrm{COOH})$ on the $\mathrm{ZnOnF}$ electrode surface. In EDC-NHS coupling chemistry, the EDC (0.2 M) works as a coupling agent while NHS (0.05 M) works as an activator. This chemical treatment allowed for the binding of the ZnOnFs and the antibody (anti-ErbB2) via a covalent amide 


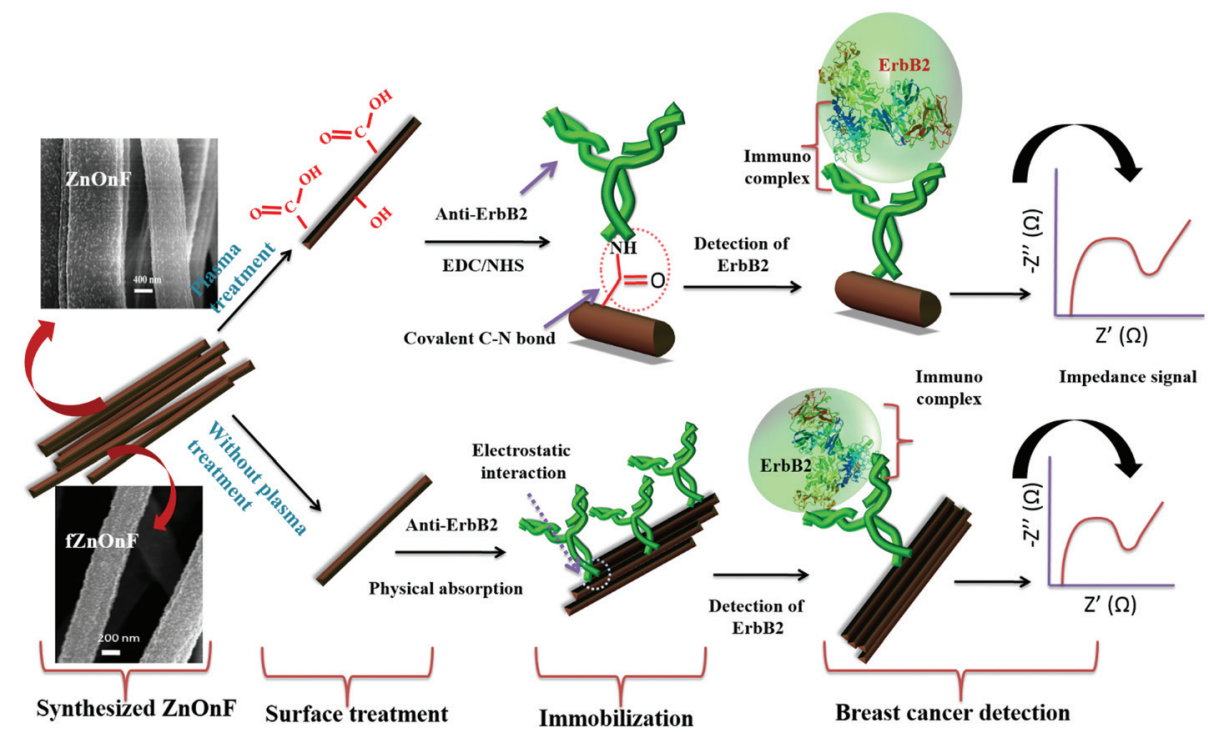

Scheme 2 Immunosensor fabrication for a label-free detection of the breast cancer biomarker.

bond on the fZnOnF (plasma treated) surface. An anti-ErbB2 solution $\left(0.02 \mathrm{mg} \mathrm{mL} \mathrm{mL}^{-1}\right)$ was spread on the $\mathrm{fZnOnF}$ surface and kept overnight under humid conditions $\left(4^{\circ} \mathrm{C}\right)$ followed by washing with phosphate buffer containing $0.05 \%$ in order to remove any unbound anti-ErbB2. The amide $(\mathrm{C}-\mathrm{N})$ bond formation between the $-\mathrm{COOH}$ groups of the fZnOnFs-ITO and the $-\mathrm{NH}_{2}$ groups of anti-ErbB2 result in improved immobilization compared to the electrostatic interactions on the $\mathrm{ZnOnF}$ surface. BSA (2 $\mathrm{mg} \mathrm{dL}^{-1}$ ) was used for blocking the nonspecific adsorption of anti-ErbB2. The specific binding (paratope) of anti-ErbB2 on the transducer surface allows its interaction with antigen ErbB2 (epitope) resulting in a change of electrochemical signal. For the electrochemical impedance studies, the fabricated electrodes were immersed in a phosphate buffer saline (PBS) solution containing ferro/ferricyanide mediator. Scheme 2 shows the fabrication of the immunosensor and conjugation of the breast cancer biomarker with the surface functionalization.

\section{Acknowledgements}

We are thankful to the Department of Science and Technology (DST), New Delhi, India for their support to the Unit on Soft Nanofabrication at the Indian Institute of Technology Kanpur. Authors thank Dr Ved V. Agrawal for interesting discussions.

\section{Notes and references}

1 A. Mishra and M. Verma, Cancers, 2010, 2, 190-208.

2 G. Orphanos and P. Kountourakis, Hematology/Oncology and Stem Cell Therapy, 2012, 5, 127-137.

3 D. Hanahan and R. A. Weinberg, Cell, 2011, 144, 646-674.
4 H. Zhang, A. Berezov, Q. Wang, G. Zhang, J. Drebin, R. Murali and M. I. Greene, J. Clin. Invest., 2007, 117, 2051-2058.

5 A. Vasudev, A. Kaushik and S. Bhansali, Biosens. Bioelectron., 2013, 39, 300-305.

6 D. $\mathrm{Yu}$ and M.-C. Hung, Breast cancer chemosensitivity, Springer, New York, USA, 2007.

7 B. V. Chikkaveeraiah, A. A. Bhirde, N. Y. Morgan, H. S. Eden and X. Chen, ACS Nano, 2012, 6, 6546-6561.

8 T. Y. Rakovich, O. K. Mahfoud, B. M. Mohamed, A. PrinaMello, K. Crosbie-Staunton, T. V. D. Broeck, L. D. Kimpe, A. Sukhanova, D. Baty, A. Rakovich, S. A. Maier, F. Alves, F. Nauwelaers, I. Nabiev, P. Chames and Y. Volkov, ACS Nano, 2014, 8, 5682-5695.

9 R. Akter, M. A. Rahman and C. K. Rhee, Anal. Chem., 2012, 84, 6407-6415.

10 K.-T. Yong, H. Ding, I. Roy, W.-C. Law, E. J. Bergey, A. Maitra and P. N. Prasad, ACS Nano, 2009, 3, 502-510.

11 J. Yao, M. Yang and Y. Duan, Chem. Rev., 2014, 114, 61306178.

12 K. Mondal, M. A. Ali, V. V. Agrawal, B. D. Malhotra and A. Sharma, ACS Appl. Mater. Interfaces, 2014, 6, 2516-2527.

13 M. Ahmad, C. Pan, Z. Luo and J. Zhu, J. Phys. Chem. C, 2010, 114, 9308-9313.

14 Y. Fang, Q. Pang, X. Wen, J. Wang and S. Yang, Small, 2006, 2, 612-615.

15 H. Hong, J. Shi, Y. Yang, Y. Zhang, J. W. Engle, R. J. Nickles, X. Wang and W. Cai, Nano Lett., 2011, 11, 3744-3750.

16 P. K. Vabbina, A. Kaushik, N. Pokhrel, S. Bhansali and N. Pala, Biosens. Bioelectron., 2015, 63, 124-130.

17 S. S. Mali, H. Kim, W. Y. Jang, H. S. Park and P. S. Patil, ACS Sustainable Chem. Eng., 2013, 1, 1207-1213.

18 J. Liu, P. Pham, V. Haguet, F. Sauter-Starace, L. Leroy, A. Roget, E. Descamps, A. Bouchet, A. Buhot, P. Mailley and T. Livache, Anal. Chem., 2012, 84, 3254-3261. 
19 M. D. Scanlon, U. Salaj-Kosla, S. Belochapkine, D. MacAodha, D. Leech, Y. Ding and E. Magner, Langmuir, 2012, 28, 2251-2261.

20 P. R. Solanki, A. Kaushik, V. V. Agrawal and B. D. Malhotra, NPG Asia Mater., 2011, 3, 17-24.

21 P. Si, S. Ding, J. Yuan, X. W. D. Lou and D.-H. Kim, ACS Nano, 2011, 5, 7617-7626.

22 C. E. Bunker, K. C. Novak, E. A. Guliants, B. A. Harruff, M. J. Meziani, Y. Lin and Y.-P. Sun, Langmuir, 2007, 23, 10342-10347.

23 H. S. Yoo, T. G. Kim and T. G. Park, Adv. Drug Delivery Rev., 2009, 61, 1033-1042.

24 X. Ren, D. Chen, X. Meng, F. Tang, X. Hou, D. Han and L. Zhang, J. Colloid Interface Sci., 2009, 334, 183-187.

25 R. S. Kane and A. D. Stroock, Biotechnol. Prog., 2007, 23, 316-319.

26 K. Mondal, S. Bhattacharyya and A. Sharma, Ind. Eng. Chem. Res., 2014, 53, 18900-18909.

27 X. Xu, B. Z. Tian, J. L. Kong, S. Zhang, B. H. Liu and D. Y. Zhao, Adv. Mater., 2003, 15, 1932-1936.

28 H. Hong, J. Shi, Y. Yang, Y. Zhang, J. W. Engle, R. J. Nickles, X. Wang and W. Cai, Nano Lett., 2011, 11, 3744-3750.

29 C. Baj-Rossi, G. D. Micheli and S. Carrara, Sensors, 2012, 12, 6520-6537.

30 S. Myung, A. Solanki, C. Kim, J. Park, K. S. Kim and K.-B. Lee, Adv. Mater., 2011, 23, 2221-2225.
31 G. Rodríguez-Gattorno, P. Santiago-Jacinto, L. RendonVázquez, J. Németh, I. Dekany and D. Diaz, J. Phys. Chem. $B, 2003$, 107, 12597-12604.

32 M. E. Aguirre, H. B. Rodríguez, E. S. Roman, A. Feldhoff and M. A. Grela, J. Phys. Chem. C, 2011, 115, 24967-24974.

33 P. Singh, K. Mondal and A. Sharma, J. Colloid Interface Sci., 2013, 394, 208-215.

34 S. Srivastava, M. A. Ali, P. R. Solanki, P. M. Chavhan, M. K. Pandey, A. Mulchandani, A. Srivastava and B. D. Malhotra, RSC Adv., 2013, 3, 228-235.

35 Z. Tehrani, G. Burwell, M. A. M. Azmi, A. Castaing, R. Rickman, J. Almarashi, P. Dunstan, A. M. Beigi, S. H. Doak and O. J. Guy, 2D Mater., 2014, 1, 025004.

36 J. T. Gohring, P. S. Dale and X. Fan, Sens. Actuators, B, 2010, 146, 226-230.

37 N. A. Mansor, Z. M. Zain, H. H. Hamzah, M. S. A. Noorden, S. S. Jaapar, V. Beni and Z. H. Ibupoto, Open Journal of Applied Biosensor, 2014, 3, 9-17.

38 M. A. Ali, S. Srivastava, M. K. Pandey, V. V. Agrawal, R. John and B. D. Malhotra, Anal. Chem., 2014, 86, 1710-1718.

39 Z. Matharu, A. J. Bandodkar, G. Sumana, P. R. Solanki, E. M. I. M. Ekanayake, K. Kaneto, V. Gupta and B. D. Malhotra, J. Phys. Chem. B, 2009, 113, 1440514412.

40 Y. Zhu, P. Chandra and Y.-B. Shim, Anal. Chem., 2013, 85, 1058-1064. 